\title{
The Fomalhaut debris disk seen from every angle with interferometry
}

O. Absil, B. Mennesson, J.-B. Le Bouquin, J.-C. Augereau, R. Millan-Gabet, et al.

O. Absil, B. Mennesson, J.-B. Le Bouquin, J.-C. Augereau, R. MillanGabet, M. Colavita, P. Hinz, W. Liu, G. Serabyn, "The Fomalhaut debris disk seen from every angle with interferometry," Proc. SPIE 7734, Optical and Infrared Interferometry II, 773417 (21 July 2010); doi: 10.1117/12.858260

SPIE Event: SPIE Astronomical Telescopes + Instrumentation, 2010, San Diego, California, United States 


\title{
The Fomalhaut debris disk seen from every angle with interferometry
}

\author{
O. Absil ${ }^{a}$, B. Mennesson ${ }^{b}$, J.-B. Le Bouquin ${ }^{c}$, J.-C. Augereau ${ }^{c}$, R. Millan-Gabet ${ }^{b}$, \\ M. Colavita ${ }^{b}$, P. Hinz ${ }^{d}$, W. Liu ${ }^{d}$ and G. Serabyn ${ }^{b}$ \\ ${ }^{a}$ IAGL, Université de Liège, 17 Allée du Six Août, B-4000 Sart Tilman, Belgium; \\ ${ }^{b}$ JPL, California Institute of Technology, 4800 Oak Grove Drive, Pasadena, CA 91109, USA; \\ ${ }^{c}$ LAOG-UMR 5571, CNRS and Université Joseph Fourier, BP 53, F-38041 Grenoble, France; \\ ${ }^{d}$ Steward Observatory, University of Arizona, 933 North Cherry Av, Tucson, AZ 85721, USA
}

\begin{abstract}
In this paper, we present the results of three different studies of the Fomalhaut debris disk with infrared interferometry. First, VLTI/AMBER measurements are used to determine the position angle of the slightly oblate rapidly rotating photosphere by means of differential phase measurements across the Br-gamma photospheric line. This measurement allows us to confirm that the debris disk is located in the equatorial plane of its host star. Second, we use VLTI/VINCI to search for resolved near-infrared emission around the stellar photosphere, which would correspond to the presence of large amounts of hot dust grains located between the sublimation radius and the habitable zone. Our observations reveal a small excess of $0.88 \% \pm 0.12 \%$ in $\mathrm{K}$ band relative to the photospheric flux. Finally, we use the Keck Interferometer Nuller in order to derive additional constraints on the nature of the resolved infrared emission. Our observations suggest a marginal detection of a circumstellar excess at $10 \mu \mathrm{m}$, which we use together with the VINCI detection to model the circumstellar emission. Preliminary results from this modeling effort are discussed.
\end{abstract}

Keywords: Circumstellar dust, planetary systems, interferometry

\section{INTRODUCTION}

At 7.7 pc from the Sun, Fomalhaut is one of the closest main sequence star surrounded by a spatially resolved debris disk. It has been studied at many different wavelengths from the visible to the sub-millimeter regime. At long wavelengths (far-infrared and beyond), the disk shows a pair of intensity maxima interpreted as the ansae of an inclined ring of about $140 \mathrm{AU}$ in radius. ${ }^{1,2}$ This particular geometry was subsequently confirmed with HST/ACS imaging, ${ }^{3}$ which also provided high accuracy estimations of the disk inclination and position angle: $i_{\text {disk }}=65.9 \pm 0.4$ and $\mathrm{PA}_{\text {disk }}=156.0 \pm 0.3$, under the assumption that the disk is intrinsically circular. In the scattered light images, dust particles are confined in a narrow ring of about $25 \mathrm{AU}$ in width, with sharp edges that suggest the presence of a planetary body at about $120 \mathrm{AU} .{ }^{4}$ It was also noted that the center of the ring is shifted by $15 \mathrm{AU}$ with respect to the central star position, pointing to still poorly understood dynamical effects that should also be related to the presence of planetary-mass or sub-stellar companions. Recently, the presence of a planetary companion at the expected orbital distance (119 AU) was confirmed with HST/ACS coronagraphic imaging, ${ }^{5}$ which further boosted the general interest in this system.

Because protoplanetary disks are mostly evacuated of their dust and gas by $10 \mathrm{Myr}$, circumstellar dust disks around main sequence stars are thought to be replenished by the populations of planetesimals that were not used to build up planets. These leftovers are supposed to produce dust by mutual collisions or cometary activity. The study of those debris disks provides one of the best means to explore the properties (size, density, orientation) and evolution of planetary systems. Although the Fomalhaut debris disk has been studied in many details during the last few years, its inner dust content remains rather elusive, with only weak constraints on a warm dust population within $20 \mathrm{AU}$ provided by Spitzer observations. ${ }^{2}$ The main challenges for characterizing this warm dust population are the small angular separation and the high contrast between the star and the inner disk.

Send correspondence to O.A. (absil@astro.ulg.ac.be)

Optical and Infrared Interferometry II, edited by William C. Danchi, Françoise Delplancke, Jayadev K. Rajagopal, Proc. of SPIE Vol. 7734,773417 • @ 2010 SPIE · CCC code: 0277-786X/10/\$18 · doi: 10.1117/12.858260 
Infrared interferometry is an appropriate tool to tackle these challenges: by providing an angular resolution as good as a few milli-arcseconds (mas), it can potentially resolve dust populations down to a fraction of an AU from Fomalhaut.

In this paper, we present the results of three different interferometric studies recently carried out on the Fomalhaut system in order to better understand its architecture. The first study aims at checking whether the resolved (cold) debris disk is located in the equatorial plane of its parent star, as predicted by standard formation theories, while the other two studies aim at charcterizing the dust content of the innermost part of the debris disk (within a few AUs).

\section{SPIN-ORBIT ALIGNMENT OF THE DISK AND THE PHOTOSPHERE}

The spin-orbit alignment of planetary systems with the photosphere of their host stars has recently been put into test in several transiting planetary systems, revealing strong misalignment for a significant fraction of hot Jupiter-type planets. With the advent of spectrally dispersed optical long baseline interferometry, it becomes possible to put this hypothesis into test also for resolved debris disks around bright rapid rotators. Indeed, the association of high spatial and high spectral resolution allows the orientation of stellar axes to be quickly (geometrically) recovered, by differential astrometry across a photospheric line enlarged by stellar rotation. Technically, the spectro-astrometric shift (i.e., the differential-phase) is measured across a photospheric line. This shift is perfectly orthogonal to the rotation axis and therefore provides an immediate estimation of its orientation.

\subsection{Observations and data analysis}

We have collected data with the AMBER instrument at the VLTI, used in K band in its Medium Resolution mode $(R \simeq 1500)$. Two triangles of baselines were used: A0-K0-G1 with ground baselines ranging from 96 to $128 \mathrm{~m}$, and D0-H0-G1 with ground baselines ranging from 64 to $72 \mathrm{~m}$. The observations of Fomalhaut were interleaved with calibration observations, for which giant stars showing no $\mathrm{Br}-\gamma$ emission line were selected. Differential phase were extracted using the amdlib package (v2.99), and consecutive observations of Fomalhaut were grouped into single data points with enhanced signal-to-noise ratio (SNR). Subtracting the instrumental phase from the scientific phase removed all fine spectral features down to a level of 0.5 (uncertainty level). However, atmospheric dispersion was not perfectly canceled out. It was efficiently removed by fitting the spectral range $2.03-2.27 \mu \mathrm{m}$ with an ad hoc polynomial law of order 3 .

The calibrated differential phases $\phi$ were converted into differential astrometric shifts $p$ using the standard formula $p=-\phi / 2 \pi \times \lambda / B$, with $B$ the baseline length and $\lambda$ the effective wavelength (Figure 1 ). This quantity represents the projection, in the baseline direction, of the $2 \mathrm{D}$ photo-center in the plane of the sky, hereafter called p. A single astrometric solution (i.e., a single $2 \mathrm{D}$ vector $\mathbf{p}$ ) was fitted to all observations available within a single spectral channel (i.e., the 12 projections $p$ ). This global fit is perfectly linear and has the advantage of showing the ultimate astrometric performance. The astrometric error ellipses were found to be almost circular thanks to the relatively uniform $u, v$ plane coverage provided by our observations. Uncertainties are in the range $\pm 2 \mu$ as to $\pm 6 \mu$ as, with typically $\pm 3 \mu$ as across the $\mathrm{Br}-\gamma$ line. It is among the most precise spectro-astrometric measurements ever achieved, if not the best.

\subsection{The Fomalhaut rotation axis}

To extract the scientifically useful information, we plot the spectro-astrometric photocenters on the plane of the sky. As a first check, we plot the photocenters in two spectral windows of continuum around the Br- $\gamma$ line (Figure 2a). The dispersion of the data points as a function of wavelength in the continuum is fully compatible with our estimated uncertainties $( \pm 3 \mu \mathrm{as})$ and shows no systematic behaviour or unexpected features at our precision level.

On the contrary, a significant astrometric displacement is detected when plotting the signal across the $\mathrm{Br}-\gamma$ line (Figure 2b). The photo-center is displaced in one direction in the blue wing of the line, and is identically displaced in the opposite direction in the red wing. The shift is compatible with zero in the center of the line, and its maximum amplitude in the line wings reaches about $15 \mu$ as. This is a clear detection of the effect of 


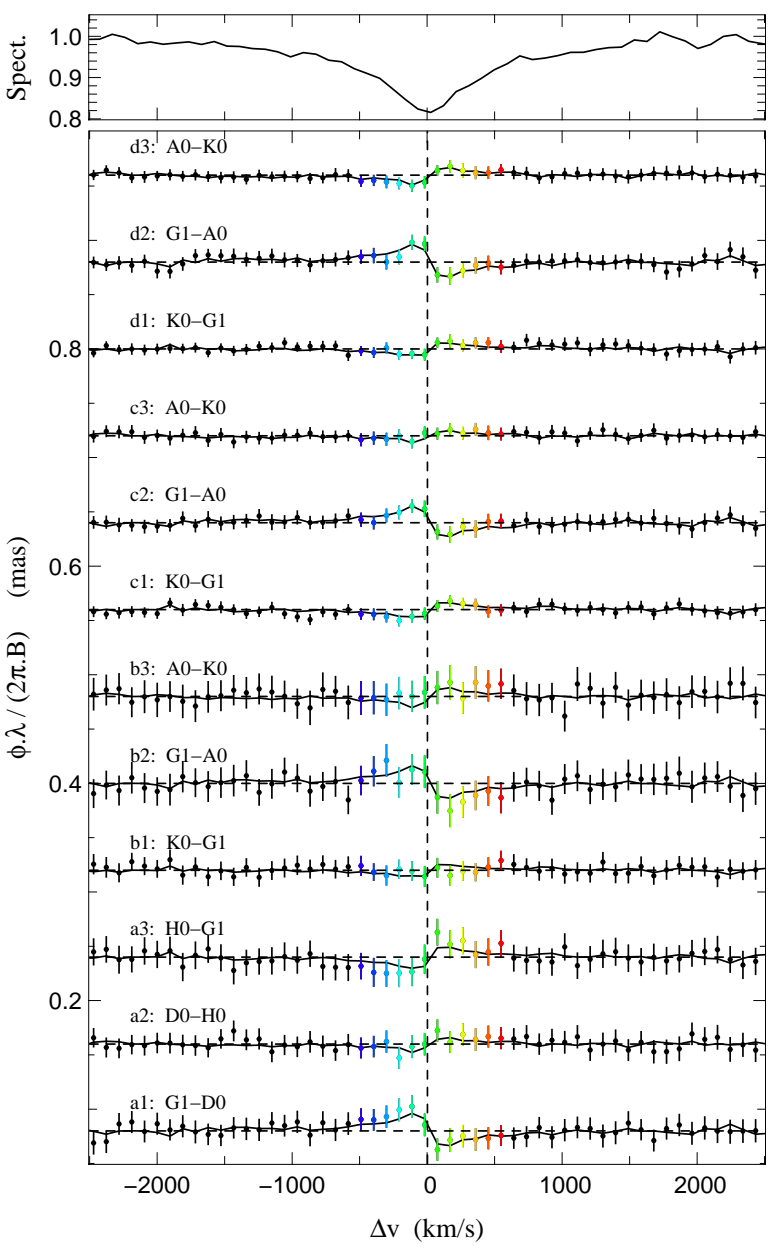

Figure 1. Top. Continuum-normalized spectrum obtained with AMBER for a spectral window centered on the Br- $\gamma$ line. Bottom. Differential phase measurements converted into spectro-astrometric shifts $p(\lambda)$ in mas. Colours represent the position within the line, from blue to red. The solid lines show the results, reprojected on the baselines, of the global 2D astrometric solution $p(\lambda)$ performed independently for each spectral channel. Phase spectra are shifted vertically by 0.08 mas.

a)

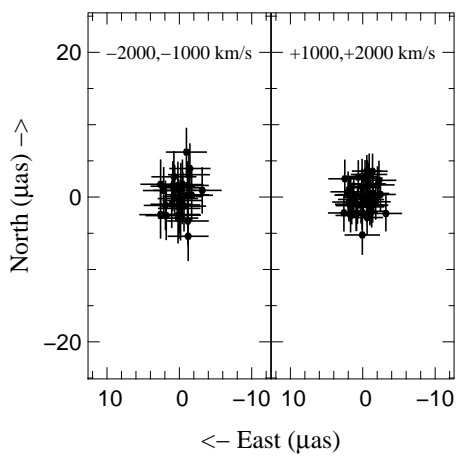

b)

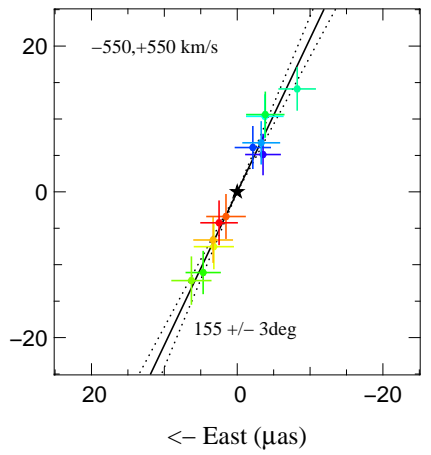

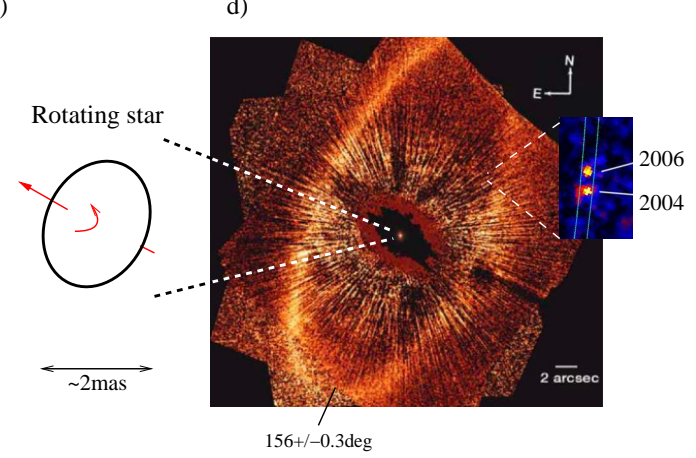

Figure 2. AMBER spectro-astrometric positions $p(\lambda)$ in the continuum (a) and across the Br- $\gamma$ absorption line (b). Colors refer to the wavelength bin, as shown in Figure 1. The signature of the rotating photosphere (c) is clearly detected and is compared to the debris disk and the planetary companion (d) imaged in the visible. ${ }^{5}$ For the sake of clarity, the astrometric error ellipses are represented by their projection in the North and East directions. 
rotation on the stellar photosphere, from which the $\mathrm{Br}-\gamma$ absorption line originates. Interpreting such a small astrometric shift does not require detailed modeling of the fast rotating atmosphere. In particular, the astrometric displacement is necessarily perpendicular to the position angle of the stellar rotation axis. Fitting our complete data set, we find the stellar rotation axis to have a position angle $\mathrm{PA}_{\star}=65^{\circ} \pm 3^{\circ}$. This value is perpendicular to the literature measurement for the disk angle $\mathrm{PA}_{\text {disk }}=156^{\circ} 0 \pm 0.3$ (Figure 2c and 2d).

Besides constraining the orientation of the stellar photosphere, spatially and spectrally resolved observations also constrain the direction of its spin vector. Taking into account that the Br- $\gamma$ line is in absorption, we conclude that the South-East part of the star is moving towards us. If we assume that the orbital angular momentum of the planetary companion points towards the same direction as the stellar spin, the Western side of the debris disk is located on the observer's side of the sky plane. Combined with the observed increased brightness in the Eastern part of the disk, ${ }^{3}$ this suggests that the dust grains are mostly backward-scattering. This finding is in contradiction to the well-known forward-scattering properties of circumstellar dust grains in our Solar system, generally assumed to be true in all debris disks. Unfortunately, this question only appeared as a very interesting by-product at the time of the study, and our observing strategy was not specifically designed to answer it. Especially, no check star is available in the data set to secure the sign of the AMBER phase.

We did our best to calibrate the phase sign a-posteriori. First we checked that a positive delay corresponds to a negative phase as measured by the AMBER reduction package we used. Secondly, we converted the phase curvature across the K-band (due to atmospheric refraction) into actual position on the sky. We found that the blue part of the band is indeed shifted toward the zenith (as it should be). However, even if we are confident, we cannot draw definite conclusions before a real spectro-astrometric reference has been observed.

\section{A K-BAND VLTI/VINCI SEARCH FOR EXOZODIACAL DUST}

The present section focuses on the search for hot circumstellar dust around Fomalhaut in the near-infrared regime using the VINCI instrument of the VLT Interferometer. The principle for warm dust detection with interferometry is based on the fact that the stellar photosphere and its surrounding dust disk have different spatial scales. For an A-type star at $7.7 \mathrm{pc}$, the angular diameter of the photosphere is about 2 mas, while the circumstellar disk extends beyond the sublimation radius of dust grains, typically located around 0.15 AU (i.e., 20 mas) for black body grains sublimating at $T_{\text {sub }} \simeq 1500 \mathrm{~K}$. The circumstellar disk is thus fully resolved at baselines as short as $10 \mathrm{~m}$ in the near-infrared and contributes as an incoherent background emission at longer baselines, while the stellar photosphere is only resolved at about $150 \mathrm{~m}$. The presence of the circumstellar disk then shows up as a decrease of visibility at baselines longer than about $10 \mathrm{~m}$ with respect to the expected visibility of the stellar photosphere. This detection method works best at short baselines where the stellar photosphere is completely unresolved.

\subsection{Observations and data reduction}

The bright star Fomalhaut has been observed on several occasions between 2001 and 2004 with VINCI, the VLT Interferometer Commissioning Instrument, which coherently combines the infrared light coming from two telescopes in the infrared $H$ and $K$ bands. ${ }^{6}$ Observations have been obtained on long baselines (B3-M0, E0-G1, $\mathrm{U} 1-\mathrm{U} 3$, all $\sim 100 \mathrm{~m}$ ), medium baselines (D0-H0, $64 \mathrm{~m}$ ) and short baselines (E0-G0, $16 \mathrm{~m}$ ) with various $u, v$ plane orientations. The observations at long and medium baselines have already been reported in two papers, ${ }^{7,8}$ while the observations at short baselines were extracted from the ESO archives. ${ }^{9}$

Most of the observations described here have been obtained with the 40-cm test siderostats of the VLTI. Their field-of-view (FOV) is limited by the use of single-mode fibers inside the VINCI instrument, and can be described by a 2D Gaussian function with a full width at half maximum (FWHM) of 1".6 in the $K$ band under standard atmospheric conditions at Cerro Paranal. This translates into a linear FOV radius at half maximum of about $6 \mathrm{AU}$ at the distance of Fomalhaut.

Data reduction was performed by using a wavelet analysis of the fringe power spectrum ${ }^{10}$ implemented in the VNDRS data reduction software. The resulting coherence factors (or raw squared visibilities) have been converted into calibrated visibilities by estimating the instrumental transfer function with interleaved observations of calibrator stars with known diameters, taking into account the wide bandwidth effect. The uncertainties on 

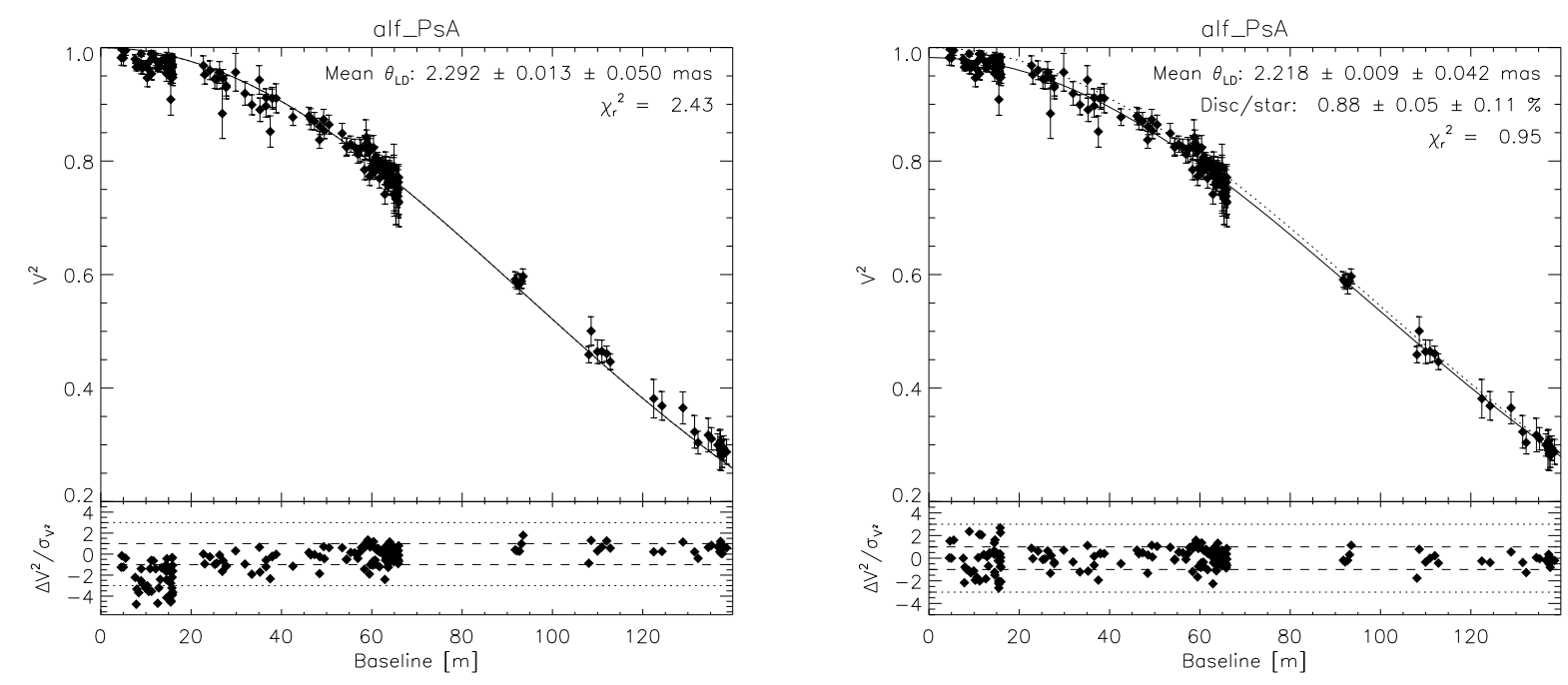

Figure 3. Left. Result of the fit of an oblate limb-darkened stellar photosphere to our full data set. For the sake of clarity, the solid line corresponds to a circular photosphere with a diameter equal to the geometric mean of the diameter of the best-fit oblate stellar model, while the residuals are computed with the full 2D photospheric model. The dashed and dotted lines in the bottom panel represent respectively a $1 \sigma$ and a $3 \sigma$ deviation with respect to the best-fit model. Right. Result of the fit of a star-disk model to our full data set. The solid line represents the best fit star-disk model, while the dotted line represents the best-fit result with a single star for comparison.

the resulting squared visibilities have been separated into statistical error (related to the dispersion of the raw visibilities) and systematic error (induced by the uncertainty on the calibrators' angular diameters).

\subsection{Evaluating the amount of circumstellar emission}

As a first step, we attempt to fit a photospheric model to our data, taking into account the expected limbdarkening and oblateness of the Fomalhaut photosphere (accepting the photospheric position angle derived in Section 2). A full description of this model can be found elsewhere. ${ }^{9,11}$ Fitting our model to the whole data set gives $\theta_{\mathrm{LD}}=2.292 \pm 0.013 \pm 0.050$ mas (Figure 3, left), where the first error bar accounts for the statistical dispersion of the data while the second error bar is related to the systematic error in the evaluation of the interferometric transfer function. This result is within $2 \sigma$ of the previous estimation, ${ }^{7}$ but the residuals of the fit and the large reduced chi square $\left(\chi_{r}^{2}=2.43\right)$ clearly demonstrate that an oblate limb-darkened photosphere cannot reproduce the data satisfactorily.

This failure urges us to increase the complexity of our model. The apparent decrement of visibility observed at short baselines and the slope in the residuals of the fit suggest that another source of emission, fully resolved by the interferometer, must be present within the interferometric field-of-view. As a first step, we assume that this resolved emission is associated with the circumstellar debris disk, and to represent its contribution we use a simple model of a diffuse source uniformly distributed across the whole field-of-view. Such a model is a good approximation provided that the circumstellar disk is fully resolved at all baselines. In Figure 3 (right), we have fitted the whole VINCI data set with a limb-darkened oblate stellar photosphere surrounded by a uniform circumstellar emission. The quality of the fit is very satisfactory, with $\chi_{r}^{2}=0.95$ and no obvious trend in the residuals, which are now nicely spread around 0 . The best-fit mean limb-darkened diameter is $\theta_{\mathrm{LD}}=2.218 \pm 0.009 \pm 0.042$ mas, while the best-fit flux ratio between the circumstellar disk and the star is $0.88 \pm 0.05 \pm 0.11 \%$. Assuming that our model is representative of the actual brightness distribution, circumstellar emission is thus detected at a $7 \sigma$ level.

Our revised estimation of the stellar limb-darkened diameter of Fomalhaut $\left(\theta_{\mathrm{LD}}=2.218 \pm 0.009 \pm 0.042 \mathrm{mas}\right)$ is within the error bar of the previous estimation of Di Folco et al. ${ }^{7}$ based on long-baseline VINCI data. The large systematic error is due to the fact that most data (at short- and mid-baseline lengths) have been calibrated 


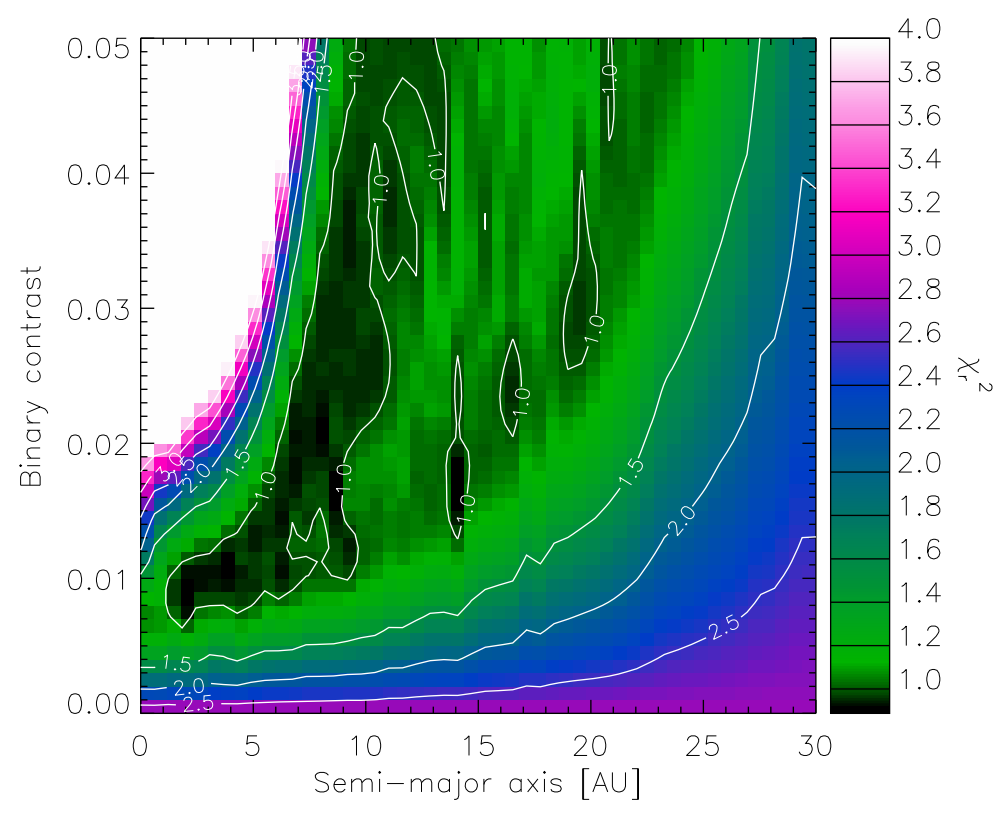

Figure 4. Reduced chi square map for the fit of a binary star model to our VINCI data, plotted as a function of the orbital semi-major axis of the companion and of the binary flux ratio. The orbital dynamics are taken into account in this model, assuming a circular orbit, and the initial orbital phase is optimized to minimize the $\chi_{r}^{2}$. We have limited the scale range to $\chi_{r}^{2} \leq 4$ for the sake of clarity. Contours are shown for $\chi_{r}^{2}$ between 1 and 3.5, with a step of 0.5 .

with the same reference star. A better accuracy on the stellar diameter can actually be obtained by fixing the disk/star contrast at $0.88 \%$ and by fitting only the long-baseline data, which have been obtained with five different calibrators. The final result is then $\theta_{\mathrm{LD}}=2.223 \pm 0.006 \pm 0.021 \mathrm{mas}\left(\chi_{r}^{2}=0.31\right)$, which is in very good agreement with the previous estimation. ${ }^{7}$ The actual angular diameter ranges between $2.246 \pm 0.022$ mas and $2.200 \pm 0.022$ mas (apparent major and minor axes), taking into account the apparent oblateness of 1.021.

Besides a circumstellar disk, another potential source of visibility deficit at short baselines would be a faint point-like object within the interferometric FOV around the target star. To reproduce the observed visibility drop, the off-axis object should have a flux ratio of about $0.88 \%$ with respect to Fomalhaut as seen through the interferometer in the $K$ band, which is equivalent to a magnitude $K=6.1$ for a close companion. Because of the Gaussian shape of the off-axis coupling efficiency into the single-mode fibers, the companion must be brighter than $K=6.1$ to reproduce the VINCI near-infrared excess if its angular separation is a significant fraction of (or larger than) the radius at half maximum $\left(00^{\prime \prime} 8\right)$ of the instrumental FOV. We estimate that the presence of such a bright background object within the instrumental FOV is very unlikely (probability $\sim 10^{-6}$ ). Therefore we only consider the case of a bound low-mass companion.

To fit our interferometric data set, we construct a model of a binary star, assuming that the companion orbits within the plane defined by the outer dust ring imaged at visible and sub-millimetric wavelengths. We therefore set the inclination and position angle of the projected orbit to 65.9 and $156^{\circ}$, respectively. In practice, coplanarity with the outer dust ring is probably not mandatory to ensure its stability, but the purpose here is only to check whether binary star models could actually fit the data rather than to explore all the possible orbital solutions. For the sake of simplicity, we further assume that the orbit is circular, so that we are left with three parameters: the semi-major axis, the orbital phase at a given time $t_{0}$ and the binary flux ratio (or contrast). Using a large number of potential values for these three parameters, we compute the position of the companion at our observing dates and the associated visibility of the binary system. We deduce the chi square between the observed visibilities and the computed ones, and for each couple semi-major axis / contrast, we search for the minimum $\chi^{2}$ as a function of the orbital phase at $t_{0}$. Thereby, we produce a $\chi^{2}$ map, which is represented in 
Fig. 4 as a function of semi-major axis and binary contrast.

The $\chi^{2}$ map shows that a whole range of semi-major axes and contrasts fit the interferometric data in a satisfactory way. The effect of the Gaussian beam profile is evident in this figure, as the binary contrast required to fit the data increases with the binary separation. We have restricted the plot range to contrasts smaller than $5 \%$, as brighter companions would most probably have been detected by simple near-infrared spectro-photometric measurements. Because the instrumental transmission becomes extremely small at linear radii larger than $30 \mathrm{AU}$ (i.e., angular separations larger than $4^{\prime \prime}$ ), binaries with larger semi-major axes cannot reproduce our interferometric data set. Conversely, for any semi-major axis smaller than about $25 \mathrm{AU}$, one can find suitable combinations of orbital parameters and flux ratio to fit the data satisfactorily (reduced $\chi^{2} \sim 1$ ). For the closest companions, located within the radius at half maximum of the FOV transmission ( 0 '! 8 , i.e., about $6 \mathrm{AU})$, the best-fit binary flux ratio is about $0.88 \%$ as expected.

In conclusion, even though our interferometric measurements span a large range of time, baseline lengths and azimuths, they are not sufficient to discriminate between a circumstellar disk and a point-like companion as the source of visibility deficit. This is mostly due to the (lack of) observing strategy, which was not optimized for such a goal. Further constraints on the possible presence of a point-like source close to Fomalhaut can however be obtained with other observing techniques, such as radial velocity and astrometric measurements, or high-contrast single-pupil imaging. The non-detections provided by these various techniques can actually be combined to reject the presence of a low-mass companion within the VLTI/VINCI field-of-view. ${ }^{9}$ We are therefore left with an extended circumstellar emission as the only plausible origin for the detected K-band excess. Among the possible sources of extended emission in the close vicinity of Fomalhaut (mostly within 0.'8), hot dust grains producing thermal emission in the near-infrared is the most straightforward scenario, since Fomalhaut is already known to be surrounded by large quantities of cold dust as well as by a warm dust population within 20 AU. ${ }^{2}$

\section{AN N-BAND KECK NULLER SEARCH FOR EXOZODIACAL DUST}

In order to further characterize the inner dust content of the Fomalhaut debris disk, we use observations obtained during the "shared risk science" and "key science" phases of the Keck Interferometer Nuller (KIN), operating with the two 10-m Keck telescope in the $\mathrm{N}$ band. The KIN nulling scheme recombines four stellar beams obtained thanks to a split mirror located just downstream of each telescope adaptive optics system. Interferometric nulling occurs separately between the two left beams, and between the two right beams. The resulting nulled output fields are then coherently recombined using a standard Michelson interferometer, called the "cross-combiner". Two different scales and baselines are then at stake: the interferometric nulling baseline separating the telescopes centers, of length $B \simeq 85 \mathrm{~m}$, and the short cross combiner baseline $b \simeq 4 \mathrm{~m}$, characteristic of the interference occurring between the "left" and "right" parts of a given telescope. The latter produces positive and negative parts in the transmission map, which is illustrated in Figure 5. This map defines the zones of the field-of-view that are transmitted or blocked by the interference process. The interferometric pattern is tapered by a gaussian field-of-view transmission created by a spatial filter downstream the beam combiner, which effectively limits the FOV to about 500 mas in radius.

\subsection{Observations and data reduction}

Fomalhaut was observed on the nights of August 28th and August 30th, 2007 (during the shared risk science observing phase of the KIN), and on the nights of July 16th and 17th, 2008 (during the key science phase). The length of the long baseline $\vec{B}$ projected onto the sky plane $\left(B_{p}\right)$ varies between $55 \mathrm{~m}$ and $82.5 \mathrm{~m}$ during the observations, while the projected baseline azimuth varies only slightly (between 47 and 50 degrees). Consequently, a constant azimuth was assumed for the data analysis and modeling. The projected length of the short baseline $\vec{b}$, on the other hand, does not change during the observations. However, its position angle changed between the 2007 and 2008 observations. It was first located at the intersection of the plane containing the line-ofsight and the zenith with the (pupil) plane perpendicular to the line-of-sight. In 2008, it was then decided to define the short baseline at the intersection of the the local horizon and the pupil plane. For relatively compact sources ( $<100$ mas in radius), the variations in the short baseline azimuth do not have a large influence on the astrophysical nulls. 


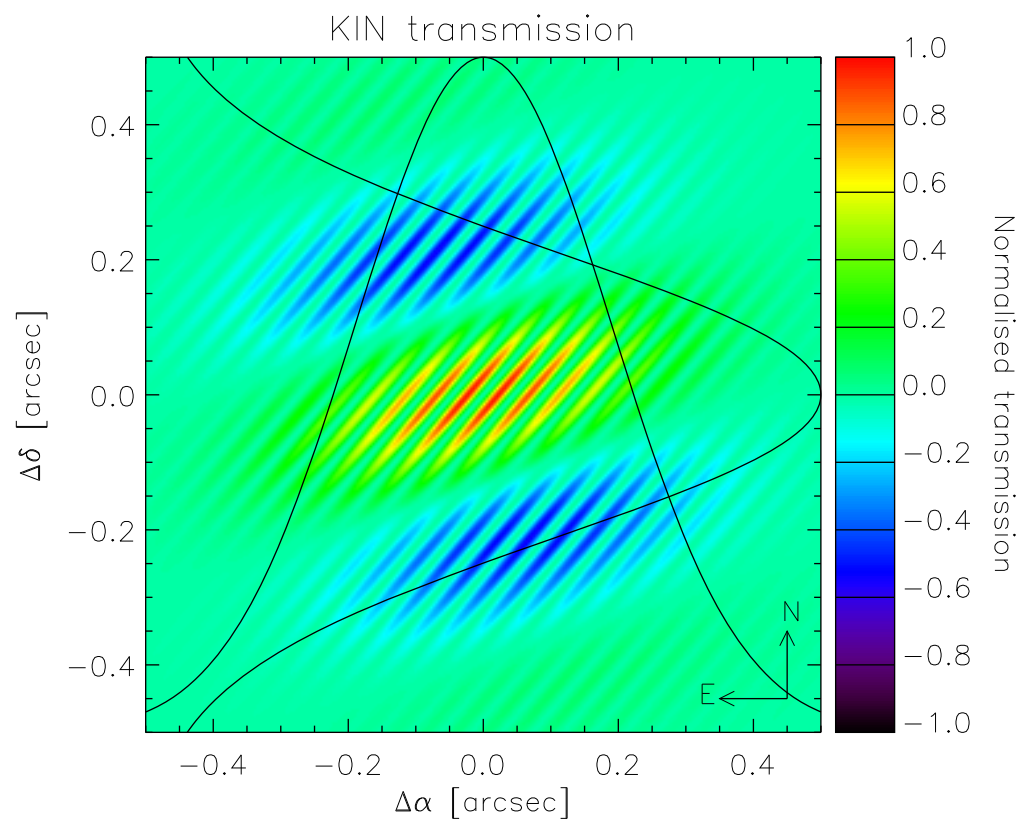

Figure 5. Keck Nuller sky transmission at $10 \mu \mathrm{m}$, when observing Fomalhaut in August 2007 on Julian date 2454342.9547 (corresponding to the average observing conditions for the 2007 observations: projected baseline $=66.62 \mathrm{~m}$, and azimuth $=49.8$ degrees). The two gaussian curves indicate the beam pattern seen by the KIN along the two sky directions.

The observations of Fomalhaut were interleaved with observations of calibrator stars, mostly consisting in K-type giants. These stars have well-known limb-darkened diameters, which we have complemented by N-band linear limb darkening coefficients, adopting the tabulated values in the literature ${ }^{12}\left(u_{N} \simeq 0.12\right.$ for $\mathrm{K}$ giants). In order to account for potential diameter or limb darkening uncertainties, all calibrators diameters error bars have been set to a conservative value of $5 \%$. The corresponding relative instrumental null depth error is $10 \%$, i.e., a $10 \mu \mathrm{m}$ null RMS uncertainty of $0.1 \%$ or smaller for the baselines and calibrator diameters considered here. The instrumental null depth at the time of a given Fomalhaut observation is then computed by interpolation between the instrumental null depths derived on adjacent calibrators. At each wavelength and for each baseline length, one can finally compute Fomalhaut's calibrated astrophysical null depth from the observed null depths.

To reduce the amount of data that needs to be used and displayed in the following discussions, we have chosen to regroup each of our two data sets into two "average" observations, computed respectively for "short" $(\sim 60 \mathrm{~m})$ and "long" $(\sim 75 \mathrm{~m})$ projected baselines. The resulting astrophysical null depths are displayed in Figure 6 (crosses with error bars). Note that the wavelength range has been limited to $8-11 \mu \mathrm{m}$ for the 2007 data set, because the data quality is not sufficient at longer wavelengths.

\subsection{Evaluating the amount of circumstellar emission}

The next step in the data analysis is to evaluate the fraction of astrophysical null that comes from the circumstellar environment of Fomalhaut. This requires to compute the null depth expected from the sole photosphere. The difference between those two quantities is noted "null excess" hereafter, and characterizes the immediate brightness distribution around Fomalhaut. To compute the photospheric null, we use Fomalhaut's limb darkened diameter $\theta_{\mathrm{LD}}=2.223 \pm 0.022$ mas presented in Section 3 and a constant linear limb darkening coefficient $u_{N}=0.06$ between 8 and $13 \mu \mathrm{m} .{ }^{12}$ The null depth expected from the sole photosphere is compared to the measured astrophysical nulls in Figure 6. Both data sets (from 2007 and 2008) show a possible null excess on top of the photospheric null, but the structure of this excess is not the exactly the same in the two data sets. In 2007 , the excess is the largest around 9.5-10 $\mu \mathrm{m}$, and seems to decrease for longer wavelengths (although the poor data quality at longer wavelengths precludes a definitive conclusion). The 2008 data set, on the other hand, 
2007

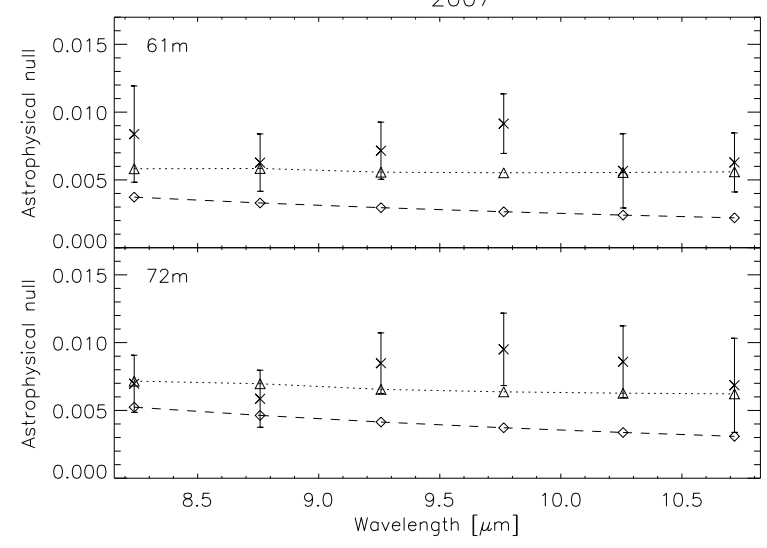

2008

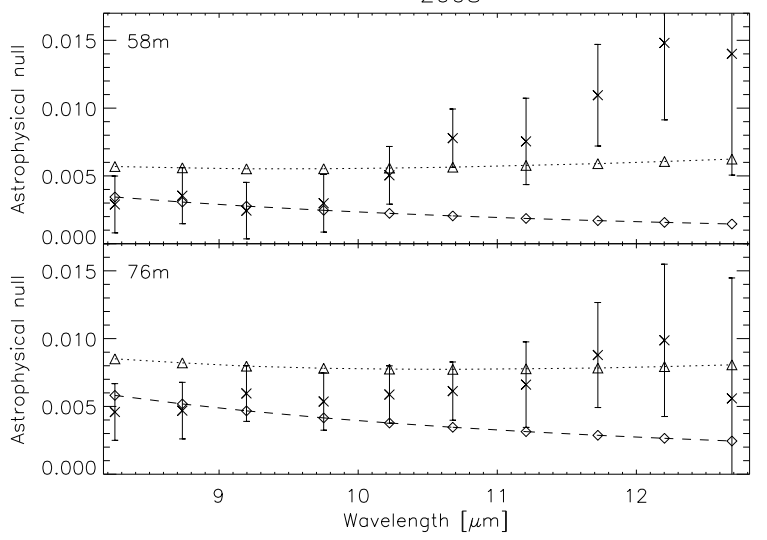

Figure 6. Left. Measured astrophysical (calibrated) null depths with error bars for Fomalhaut on "short" (top) and "long" (bottom) projected baselines in August 2007. The diamonds and dashed lines show the expected null depths for the sole stellar photosphere. The triangles and dotted lines show the expected null for the photosphere surrounded by a 250 -zodi disk. Right. Same figures for the data of July 2008.

shows no excess up to about $10.5 \mu \mathrm{m}$, where the measured nulls start to diverge from the expected photospheric null. Although the error bars are larger in the 11-13 $\mu \mathrm{m}$ region, a significant excess is still observed (especially for the short baseline). While the 2007 and 2008 data sets suggest slightly different spectral shapes for the nulls between the two epochs, it must be noted that the difference is not larger than $1 \sigma$, so that they can be considered as consistent.

\section{DISCUSSION}

In this section, we use the observations described here above to constrain the exozodiacal dust content in the Fomalhaut inner disk (implicitly accepting that the presence of hot dust grains is the most probable origin for the detected excesses). We first discuss the VINCI and KIN constraints separately, and then attempt a joint modeling of the two data sets.

\subsection{Constraints on the exozodiacal dust density}

The VINCI data provide us basically with only one parameter: the K-band flux ratio between the circumstellar emission and the stellar photosphere. We can use this information together with a model of Fomalhaut exozodiacal cloud to estimate the amount of dust. A possibility is be to use the model of the solar zodiacal dust could as seen from outside the solar system. ${ }^{13}$ The only scaling that we apply to this model concerns the dust temperature, which is increased to account for the actual photospheric temperature of Fomalhaut. In this case, we have to multiply the solar system zodiacal cloud density by 5000 to account for the K-band contrast of $0.88 \%$ between Fomalhaut and its exozodiacal cloud.

Now, we can try to use the same model can be used to reproduce the KIN data. Since the only parameter is the overall disk density, we attempt to find the most suitable exozodi density to reproduce the four data sets (2007 and 2008, short and long baselines). In Figure 6, we show that a 250-zodi disk produces a null excess in relatively good agreement with all data sets. The only significant failure of our model is to reproduce the slope in the 2008 data set, where the excess increases with wavelength. The only way to reproduce such a slope would be to place the circumstellar matter at orbital distances larger than about 1.5 AU, so that the disk to star flux ratio increases significantly from 8 to $13 \mu \mathrm{m}$. This increase in the flux ratio actually combines with the structure of the KIN transmission map (with negative parts appearing at about 150 mas, i.e., 2 AU at the distance of Fomalhaut, see Figure 5) to reinforce the increase in null excess as a function of wavelength: while at $8 \mu \mathrm{m}$ a large part of the circumstellar emission falls in a negative part of the transmission map, the increase in fringe spacing at $13 \mu \mathrm{m}$ makes the circumstellar emission fall mostly in the central, positive part of the transmission map. We note however that dust located beyond 1.5 AU cannot produce a significant K-band emission due to 
its limited temperature ( $<700 \mathrm{~K}$ for blackbody dust grains). For this reason, we mostly focus on dust within $1 \mathrm{AU}$ in the following discussion.

\subsection{Joint modeling of the VINCI and KIN data}

We have performed preliminary attempts to fit the VINCI and KIN data simultaneously using the debris disk model of Augereau et al. ${ }^{14}$ The combination of resolved observations at two different wavelengths put strong constraints on the possible location of dust grains. Producing a significant K-band excess with a modest Nband excess requires most of the grains to be hot $(\sim 1500 \mathrm{~K}$ or higher, depending on their actual sublimation temperature). The direct consequence is that most of the grains should be located close to the sublimation radius, either arranged in a narrow ring or with a steep density power law. In order to increase the sublimation temperature, highly refractory grains such as carbons are preferred over "classical" silicates. This also prevents from obtaining a large silicate emission feature around $10 \mu \mathrm{m}$.

The same kind of result had already been obtained in the case of Vega, ${ }^{15}$ where K- and N-band constraints were also both available. The physical meaning of such a model is however questionable, as small dust grains located close to the sublimation radius should not survive more than a few years due to the intense radiation pressure exerted by the stellar photosphere. One must also note that the slope of the null excess with respect to wavelength cannot be reproduced with such a model, so that a more complex model probably needs to be used.

\section{ACKNOWLEDGMENTS}

O. A. acknowledges the financial support of F.R.S.-FNRS (Postdoctoral Researcher), and support from "Communauté Française de Belgique - Actions de Recherche Concertées - Académie universitaire Wallonie-Europe". This research was partly funded by the International Space Science Institute ("Exozodiacal Dust Disks and Darwin" working group). Part of this work is based on observations collected at the VLTI (ESO Paranal, Chile), under program ID 082.C-0376 (AMBER Guaranteed Time of the Osservatorio Astrofisico di Arcetri, INAF, Italy) and using public VINCI commissioning data. Some of the data presented herein were obtained at the W. M. Keck Observatory, which is operated as a scientific partnership among the California Institute of Technology, the University of California, and the National Aeronautics and Space Administration. The Observatory was made possible by the generous financial support of the W. M. Keck Foundation.

\section{REFERENCES}

[1] Holland, W. S., Greaves, J. S., Zuckerman, B., Webb, R. A., McCarthy, C., Coulson, I. M., Walther, D. M., Dent, W. R. F., Gear, W. K., and Robson, I., "Submillimetre images of dusty debris around nearby stars," Nature 392, 788-791 (Apr. 1998).

[2] Stapelfeldt, K. R., Holmes, E. K., Chen, C., Rieke, G. H., Su, K. Y. L., Hines, D. C., Werner, M. W., Beichman, C. A., Jura, M., Padgett, D. L., Stansberry, J. A., Bendo, G., Cadien, J., Marengo, M., Thompson, T., Velusamy, T., Backus, C., Blaylock, M., Egami, E., Engelbracht, C. W., Frayer, D. T., Gordon, K. D., Keene, J., Latter, W. B., Megeath, T., Misselt, K., Morrison, J. E., Muzerolle, J., Noriega-Crespo, A., Van Cleve, J., and Young, E. T., "First Look at the Fomalhaut Debris Disk with the Spitzer Space Telescope," ApJS 154, 458-462 (Sept. 2004).

[3] Kalas, P., Graham, J. R., and Clampin, M., "A planetary system as the origin of structure in Fomalhaut's dust belt," Nature 435, 1067-1070 (June 2005).

[4] Quillen, A. C., "Predictions for a planet just inside Fomalhaut's eccentric ring," MNRAS 372, L14-L18 (Oct. 2006).

[5] Kalas, P., Graham, J. R., Chiang, E., Fitzgerald, M. P., Clampin, M., Kite, E. S., Stapelfeldt, K., Marois, C., and Krist, J., "Optical Images of an Exosolar Planet 25 Light-Years from Earth," Science 322, 1345(Nov. 2008).

[6] Kervella, P., Gitton, P. B., Segransan, D., di Folco, E., Kern, P. Y., Kiekebusch, M., Duc, T. P., Longinotti, A., Coude du Foresto, V., Ballester, P., Sabet, C., Cotton, W. D., Schoeller, M., and Wilhelm, R., "VINCI, the VLTI commissioning instrument: status after one year of operations at Paranal," in [Interferometry in Optical Astronomy II], Traub, W., ed., Proc. SPIE 4838, 858-869 (Feb. 2003). 
[7] Di Folco, E., Thévenin, F., Kervella, P., Domiciano de Souza, A., Coudé du Foresto, V., Ségransan, D., and Morel, P., "VLTI near-IR interferometric observations of Vega-like stars," A\&\&A 426, 601-617 (2004).

[8] Le Bouquin, J.-B., Labeye, P., Malbet, F., Jocou, L., Zabihian, F., Rousselet-Perraut, K., Berger, J.-P., Delboulbé, A., Kern, P., Glindemann, A., and Schöller, M., "Integrated optics for astronomical interferometry. VI. Coupling the light of the VLTI in K band," $A \mathscr{E} A$ 450, 1259-1264 (May 2006).

[9] Absil, O., Mennesson, B., Le Bouquin, J.-B., Di Folco, E., Kervella, P., and Augereau, J.-C., "An interferometric study of the Fomalhaut inner debris disk I. Near-infrared detection of hot dust with VLTI/VINCI," ApJ 704, 150-160 (Oct. 2009).

[10] Kervella, P., Ségransan, D., and Coudé du Foresto, V., "Data reduction methods for single-mode optical interferometry. Application to the VLTI two-telescopes beam combiner VINCI," $A \& A$ 425, 1161-1174 (2004).

[11] Absil, O., Di Folco, E., Mérand, A., Augereau, J.-C., Coudé Du Foresto, V., Defrère, D., Kervella, P., Aufdenberg, J. P., Desort, M., Ehrenreich, D., Lagrange, A.-M., Montagnier, G., Olofsson, J., Ten Brummelaar, T. A., McAlister, H. A., Sturmann, J., Sturmann, L., and Turner, N. H., "A near-infrared interferometric survey of debris disc stars. II. CHARA/FLUOR observations of six early-type dwarfs," A\&A 487, 1041-1054 (Sept. 2008).

[12] Tango, W. J. and Davis, J., "The accurate determination of stellar angular diameters using broad-band stellar interferometry," MNRAS 333, 642-648 (July 2002).

[13] Kelsall, T., Weiland, J. L., Franz, B. A., Reach, W. T., Arendt, R. G., Dwek, E., Freudenreich, H. T., Hauser, M. G., Moseley, S. H., Odegard, N. P., Silverberg, R. F., and Wright, E. L., "The COBE Diffuse Infrared Background Experiment Search for the Cosmic Infrared Background. II. Model of the Interplanetary Dust Cloud," ApJ 508, 44-73 (Nov. 1998).

[14] Augereau, J. C., Lagrange, A. M., Mouillet, D., Papaloizou, J. C. B., and Grorod, P. A., "On the HR 4796 A circumstellar disk," A\& A 348, 557-569 (Aug. 1999).

[15] Absil, O., Di Folco, E., Mérand, A., Augereau, J.-C., Coudé du Foresto, V., Aufdenberg, J. A., Kervella, P., Ridgway, S. T., Berger, D. H., ten Brummelaar, T. A., Sturmann, J., Strumann, L., Turner, N. H., and McAlister, H. A., "Circumstellar material in the Vega inner system revealed by CHARA/FLUOR," A\&A 452, 237-244 (2006). 